\title{
Current Applications and Future Perspectives of CRISPR-Cas9 for the Treatment of Lung Cancer
}

\author{
Markeshaw Tiruneh G/Medhin' \\ Endeshaw Chekol Abebe $\mathbb{I D}^{2}$ \\ Tekeba Sisay ${ }^{3}$ \\ Nega Berhane ${ }^{3}$ \\ Tesfahun Bekele Snr' \\ Tadesse Asmamaw Dejenie (iD) \\ 'Department of Biochemistry, School of \\ Medicine, College of Medicine and Health \\ Sciences, University of Gondar, Gondar, \\ Ethiopia; ${ }^{2}$ Department of Biochemistry, \\ College of Health Sciences, Debre Tabor \\ University, Debre Tabor, Ethiopia; \\ ${ }^{3}$ Institute of Biotechnology, College of \\ Natural Science, University of Gondar, \\ Gondar, Ethiopia
}

\begin{abstract}
Clustered Regularly Interspaced Short Palindromic Repeats and CRISPR-associated proteins are referred to as CRISPR-Cas9. Bacteria and archaea have an adaptive (acquired) immune system. As a result, developing the best single regulated RNA and Cas9 endonuclease proteins and implementing the method in clinical practice would aid in the treatment of diseases of various origins, including lung cancers. This seminar aims to provide an overview of CRISPR-Cas9 technology, as well as current and potential applications and perspectives for the method, as well as its mechanism of action in lung cancer therapy. This technology can be used to treat lung cancer in two different ways. The first approach involves creating single directed RNA and Cas9 proteins and then distributing them to cancer cells using suitable methods. Single directed RNA looks directly at the lung's mutated epidermal growth factor receptor and makes a complementary match, which is then cleaved with Cas9 protein, slowing cancer progression. The second method is to manipulate the expression of ligand-receptors on immune lymphocytic cells. For example, if the CRISPR-Cas9 system disables the expression of cancer receptors on lymphocytes, it decreases the contact between the tumor cell and its ligand-receptor, thus slowing cancer progression.
\end{abstract}

Keywords: CRISPR, Cas9, CRISPR-Cas9 technology, cancer, lung cancer, cancer treatment

\section{Background}

The word CRISPR-Cas9 refers to Clustered Regularly Interspaced Short Palindromic Repeats and CRISPR-associated proteins. ${ }^{1-5}$ CRISPR-Cas9 system is a kind of acquired immunity possessed by most bacteria and archaea (prokaryotes) to act against their enemies (bacteriophages). ${ }^{4,6}$ It is a ribonucleic acid (RNA) guided, convenient, and versatile endonuclease platform for site-specific genome editing, ${ }^{1,7,8}$ which can play a tremendous role in the application of cancer therapy. ${ }^{1}$ The application of this technology can be used to resolve mutations and to introduce site-specific therapeutic genes in human cells so that, correcting disease-causing mutations, and alleviate disease-related symptoms. This system is also a useful tool for delineating molecular mechanisms involving hematological malignancies. ${ }^{4}$ Sequence-specific gene editing using CRISPR-Cas9 shows promise as a novel therapeutic approach for the treatment of a variety of diseases that currently lack effective treatments like cancers. ${ }^{3,9}$ To accomplish its task, it requires Cas 9 DNA endonuclease protein and single guided RNA ( $\operatorname{sgRNA}$ ) that can produce precise gene matching for editing and correction techniques. ${ }^{2}$ So the system has enabled easy manipulation of genes for the scientific community by making the hybrid to the target sequence and cleaving the double-strand DNA. ${ }^{10}$

Additionally, the CRISPR-Cas9 technology is increasingly feasible to overcome drug resistance in breast cancer therapy and will become an essential tool for personalized 
medicine. ${ }^{4}$ It is a technological breakthrough that facilitates the ability to change nucleic acids, ${ }^{11}$ and with continued improvement in the function, the system can help to develop best treatment options to a variety of genetic disease which affects several tissues in our body. ${ }^{12}$ Gene manipulation using CRISPR-Cas9 system has revolutionized and made it easy to study the work of genes and importantly opens the new era of treatment mechanisms for different disease conditions including cancer. ${ }^{13}$ Technologies like this are a simple and efficient method of targeting the required DNA regions. ${ }^{14}$ Thus, scientists have designed two main components of the system for easy detection and alteration of gene function one component is a protein Cas9 that enzymatically cleave the desired gene and the sgRNA which scans and determines where the gene of interest will be cleaved by Cas9 protein. ${ }^{3,12,15}$ The system has been scientifically optimized and developed to regulate expression of the gene, modify and edit the desired locus and this makes the technology of choice seen by the scientific community to treat or edit disease-causing mutations more efficiently than ever before. Furthermore, its application is encouraging for more vigorous gene therapy in clinical setups. ${ }^{16}$ Based on the discovery, there are three main types of CRISPR-Cas9 system (I to III) and with three additional types (IV to VI) being identified more recently. ${ }^{17}$ They are different during the processes of immunity, adaptation, expression, and interference, each type acts in distinct mechanisms to ensure genetic manipulation. Type I employs a large complex of Cas9 proteins with distinct helicase and DNase activities, while type III employs repeat-associated mysterious proteins, which form a large Cas9 superfamily. Another classification is based on subunit effectors, with multi-subunit effector complexes being the most common. Types I, III, and IV are grouped in class 1 . Those systems, on the other hand, that have a single subunit effector are categorized as class 2 comprising types II, V, and VI. ${ }^{17,18}$ Type II uses only a Single protein (Cas9) for its nuclease activity and has got more attention and adopted for genome engineering. ${ }^{5,17,19}$ Thus, the objective of this seminar is to introduce CRISPR-Cas9 technology and describe current applications and future perspectives of the system with its mechanism of action on lung cancer therapy.

\section{Components of CRISPR-Cas9, Maturation, and Spacer Acquisition}

The tracrRNA gene will be transcribed to tracrRNA, the crRNA gene will be transcribed to pre crRNA, and the Cas9 gene will be transcribed to Cas9 messenger RNA and converted to Cas9 protein, all of which will be post-transcribed and chopped off to form the mature CRISPR-Cas9 complex. ${ }^{20}$ Cas1 and Cas2 integrase, which are present in all CRISPR forms, catalyze spacer integration on the CRISPR array especially on the leader end of the repeat there will be a nucleophilic attack of the $3^{\prime} \mathrm{OH}$ of the protospacers followed by the same practice on the spacer end of the repeat. ${ }^{21}$

\section{Formulation Strategy and Delivery Mechanism}

\section{Formulation Strategy}

The CRISPR-Cas9 method has a variety of formulation methods for genome editing. The use of a plasmid-based CRISPR-Cas9 system encoding both the Cas9 protein and sgRNA from the same vector, which is necessary to avoid multiple transfections of different components of the technology, is the leading and possibly the easiest technique. The Cas9 protein and sgRNA will be expressed in the vector, which will form the sgRNA-Cas9 complex within cells to edit the target genomic sequences. ${ }^{3,12,15,18}$ The second approach involves combining Cas9 mRNA and sgRNA. The sgRNA-Cas9 complex will be formed when Cas9 mRNA is converted into Cas9 protein in cells. The third strategy is to deliver the in vitro assembled sgRNA-Cas9 complex directly to the cell. ${ }^{18}$

\section{Delivery Mechanism}

It is difficult to transmit nucleic acid in general, and CRISPR-Cas9 in particular, to the target tissue or cell. Physical and vector (viral or non-viral) approaches are two of the most widely used distribution strategies. ${ }^{11,22,23}$ Electroporation and microinjections are used in physical methods, while viral delivery strategies such as adeno associated virus (AAV) are widely used in vector-based methods since they are not disease-causing agents and can infect both dividing and non-dividing cells ${ }^{25}$ and lentivirus with inactivated integrase enzymes are under investigation. ${ }^{24}$

Another technique is lipofection (lipid-mediated nanoparticle transfection), which is possibly the most efficient CRISPR-Cas9 in vivo delivery method. ${ }^{22}$ This technique was further developed by ${ }^{26}$ and is currently being tested in clinical trials. ${ }^{13,24}$

\section{The Application of the CRISPR- Cas9 System and Its Mode of Action}

The CRISPR-Cas9 system, as discussed, a little earlier, is made up of two main components that work together to accomplish its goal. ${ }^{19}$ The sgRNA contains crRNA, which 
scans and identifies the target DNA sequences that must be cleaved and corrected, and transactivated crRNA (tracrRNA), which recruits component two, the Cas9 protein DNA endonuclease, which can sense, identify, and establish site-specific double-strand DNA breaks (DSB). ${ }^{15}$ Because of its simplicity and convenience, the bacterial type II CRISPR-Cas9 system has been used for RNA-guided engineering nucleases. ${ }^{4,18}$ However, the proto-spacer adjacent motifs (PAM) sequences are required by the method. After recognition, two Cas9 domains cleave double-stranded DNA: the endonuclease domain named for characteristic histidine and asparagine residues $(\mathrm{HNH})$ domain, which cleaves the complementary strand, and the endonuclease domain named for an E. coli protein involved in DNA repair (RuvC-like) domain, which cleaves the non-complementary strand. ${ }^{17}$ As a result, the host DNA repair machinery introduces numerous mutations such as substitutions, deletions, and insertions in the target genome, including non-homologous end joining (NHEJ) or homologous-dependent repair (HDR). ${ }^{15-18}$ Another paper, CRISPR-Cas9 for Cancer Therapy: Hopes and Challenges, supports this theory by demonstrating that the sgRNA-Cas9 complex scans and anneals to the genomic target sequence with base-pairing complementarity and precisely cleaves double-stranded DNA of the target cell after identification of the protospacer adjacent motif (PAM) sequence adjacent to the target sequence. NHEJ or HDR pathways are activated as a result of double-strand breaks. NHEJ is an error-prone repair mechanism that results in indels (insertions or deletions) of random base pairs disrupting the target sequence in the absence of a homologous repair prototype with more specific repair mechanisms. ${ }^{23,27}$

\section{Specific Application of CRISPR-Cas9 System for Treatment of Lung Cancer}

Lung cancer is the major cause of death in the United States and a significant public health concern worldwide. ${ }^{5}$ In both developed and developing countries, it is a common cause of morbidity and mortality. ${ }^{28}$ According to a study conducted by the American Lung Cancer Society in 2015, lung cancer claims the lives of almost 150,000 people each year. However, surgery and radiation were used as treatment options. The treatment was later changed to selective Tyrosine kinase inhibitors (TKIs) like gefitinib and erlotinib to inhibit the tyrosine kinase activity of epidermal growth factor receptor, which has technical difficulties and nonspecific cytotoxicity (EGFR). ${ }^{29,30}$ Extracellular ligand binding, transmembrane, and intracellular tyrosine kinase domains are found in this membrane glycoprotein. When the ligand activator binds to the extracellular ligand domain, it transduces and initiates intracellular kinase activities, which cause cellular proliferation, neovascularization, invasion, and metastasis, as well as reduced apoptosis and glycolysis activation. These medications, however, have encountered drug resistance. ${ }^{28,29}$

The CRISPR-Cas9 device is the start of a new biotechnological era and a groundbreaking technology that is being used to treat lung cancer. ${ }^{6,29}$ The system works in two ways. The first is by designing sgRNA that looks for the mutated EGFR sequence, which is then accompanied by Cas9 protein. To do so, scientists created a CRISPR device that has complementary sequences with the mutated EGFR and introduced it into the patient, as mentioned earlier which has complementary sequences with the mutated EGFR and introducing this into the patient. As this complementary sequence binds to the mutated EGFR, the Cas9 protein (endonuclease) creates a double-stranded or singlestranded DNA break, depending on the type of enzyme used, followed by DNA repair mechanisms such as homologous or non-homologous DNA repair. ${ }^{29}$ If the receptor mutation is limited, there will be no contact between the ligand activator, resulting in no cell proliferation, neovascularization, or cancer metastasis, and the problem will be solved. The inhibition of EGFR by CRISPR-Cas9 increases the expression of major histocompatibility complex class I, which improves cytotoxic lymphocyte recognition and lysis of tumor cells. ${ }^{30,31}$ Off-target effects, which can induce genome instability, gene functional disturbances, and epigenetic alterations, are a challenge. Off-target effects of CRISPR-Cas9 systems, particularly when used for therapeutic purposes, should be minimized and precisely profiled. Off-target effects are separated into two categories: off-target binding and off-target cleavage. Cas9 can bind to target sequences that are partially complementary to sgRNA and inhibit target gene transcription without cleaving them. ${ }^{8}$ Off-target binding effects may thus be removed in traditional off-target identification approaches, such as using in vitro assembled sgRNA with a long-lasting association with cas9, which also has a high proportion of on-target and high efficiency for genome editing. Another technique is to use a Cas9 variant or modified Cas 9 that can generate a single nick at one strand. ${ }^{23}$ So that the off-target effect is reduced. 
The second, and equally significant, strategy for using this biotechnological method to treat lung cancer is to search for immune cells like lymphocytes. T cells are immune cells that are extracted from the blood of patients engaged in a clinical trial for lung cancer treatment in China, and then CRISPR-Cas9 is used to knock out a gene in the cells that encodes a protein called PD-1. The edited gene cells would then be propagated in the lab before being injected back into the patient's bloodstream. ${ }^{6,25}$ Scientists took blood from the patient and extracted lymphocytes, which were then treated with a CRISPR-Cas9 gene-editing system containing a sgRNA sequence with a pattern identical to lymphocytes' programmable death 1 protein (PD 1). When the system detects its target sequence, cas9 would sever the DNA, which is then repaired by cell repair mechanisms. When the expression of the PD 1 gene is blocked or disabled, cancerous cells lack the receptor on immune lymphocytes. ${ }^{6,25}$ As a result, if lymphocytes do not express the PD 1 receptor well, there will be less contact between the cancerous ligand and receptor, causing the $\mathrm{T}$ cell receptor to identify the problematic cell and perform its function. Naturally, these manipulated lymphocytes were screened for viability and lympho-proliferation to rule out new mutations, and only those cells that passed the test were returned to the patient. ${ }^{6,25}$ Furthermore, knocking out the PD-1 protein on immune cells is necessary for caspase activation, which is needed for programmed cell death and enhanced apoptosis in cancerous cells. ${ }^{31}$ It also concludes that PD-1-deficient cells have potent antitumor activity of cytotoxic lymphocytes. The hyperactivity of the manipulated $\mathrm{T}$ cells is one of the technology's drawbacks for use in this way ${ }^{6}$ and obtaining a safe and efficient delivery method, as well as some side effects Patients with advanced NSCLC with positive PD-1 expression were assigned to a Phase I clinical trial to assess the safety of CRISPR-Cas9-mediated knockout of PD-1 gene therapy in patients with metastatic non-small cell lung cancer. Nine patients were enrolled, and eight patients received PD-1 deficient $\mathrm{T}$ cell therapy, and the patients were manifested with PD-1 deficient T cell therapy. ${ }^{25}$ Patients undergoing PD-1 deficient $\mathrm{T}$ cell therapy, on the other hand, appeared to be healthy, and researchers recommended that broader studies be conducted to determine the most appropriate dosage and immune response.

\section{Future Perspective}

In cancer biology, the CRISPR-Cas9 device has a bright future ahead of it, ${ }^{9}$, because it is a technology that is adaptable, simple, convenient and efficient. ${ }^{32,33}$ The method introduces a novel approach to cancer treatment by allowing for modifications to the genome of target cells, which was previously difficult to achieve. ${ }^{34-36}$ the technology's versatility, effectiveness, and flexibility would make it the best form of cancer care in the future. ${ }^{4,37,38}$ It will affect cancer biology as a whole in the future, ${ }^{34}$ and if researchers have devised well-organized strategies and instruments for delivering the technology to the target cell or tissue, as well as effective methods and instructions for controlling and eliminating the technology's off-target effects.

\section{Conclusion}

The CRISPR-Cas9 device is a recent biotechnological breakthrough and scientific achievement. This technology has created a new treatment option for diseases of various origins, such as cancer and infectious disease. To solve the problem, the best sgRNA must be designed using a CRISPR tool (http://crispr.mit.edu) and its associated endonuclease cas9 protein against the target sequence. However, ethical concerns, the need for the best delivery strategies, and the risk of off-target effects are only a few of the problems that must be addressed. Since the technology is still in its infancy, researchers must devise simple methods and mechanisms to track and test its protection and efficacy. For a simple comparison, the benefits of this technology are simple, fast, relatively effective, relatively precise, and versatile, while the drawbacks are distribution is difficult, ethical problems are highly conservative, some off-target effects, and some adverse effects.

\section{Abbreviations}

ATP, Adenosine triphosphate; CRISPR, Clustered regularly interspaced short palindromic repeat; CRISPR-Cas, Clustered regularly interspaced short palindromic repeatassociated; CrRNA, Clustered regularly interspaced short palindromic repeat ribonucleic acid; DNA, Deoxyribonucleic acid; DSB, Double-stranded break; EGFR, epidermal growth factor receptor; HDR, Homologous directed repair; mRNA, Messenger ribonucleic acid; NHEJ, Non-homologous end-joining; PD 1, Programmable death protein 1; RNA, Ribonucleic acid; SgRNA, Single guided ribonucleic acid; TracrRNA, Trans activating clustered regularly short palindromic repeat ribonucleic acid; TKIs, Tyrosine kinase inhibitors.

\section{Author Contributions}

All authors made substantial contributions to conception and design, acquisition of data, or analysis and interpretation of 
data; took part in drafting the article or revising it critically for important intellectual content; agreed to submit to the current journal; gave final approval of the version to be published; and agree to be accountable for all aspects of the work".

\section{Funding}

There is no funding to report.

\section{Disclosure}

The authors declare that they have no conflicts of interest for this work.

\section{References}

1. Liu T, Shen JK, Li Z, Choy E, Hornicek FJ, Duan Z. Development and potential applications of CRISPR-Cas9 genome editing technology in sarcoma. Cancer Lett. 2016;373(1):109-118. doi:10.1016/j. canlet.2016.01.030

2. Yin H, Song CQ, Dorkin JR, et al. Therapeutic genome editing by combined viral and non-viral delivery of CRISPR system components in vivo. Nat Biotechnol. 2016;34(3):328-333. doi:10.1038/ nbt. 3471

3. Zhen S, Li X. Oncogenic human papillomavirus: application of CRISPR/Cas9 therapeutic strategies for cervical cancer. Cell Physiol Biochem. 2017;44(6):2455-2466. doi:10.1159/000486168

4. Chen Y, Zhang Y. Application of the CRISPR/Cas9 system to drug resistance in breast cancer. Adv Sci. 2018;5(6):1700964. doi:10.1002/ advs.201700964

5. Shen Q, Li J, Mai J, et al. Sensitizing non-small cell lung cancer to BCL-xL-targeted apoptosis. Cell Death Dis. 2018;9(10):1-3. doi:10.1038/s41419-018-1040-9

6. Castillo A. Gene editing for the treatment of lung cancer (CRISPR-Cas9). Colomb Med. 2016;47(4):178-180. doi:10.25100/ cm.v47i4.2856

7. Platt RJ, Chen S, Zhou Y, et al. CRISPR-Cas9 knockin mice for genome editing and cancer modeling. Cell. 2014;159(2):440-455. doi:10.1016/j.cell.2014.09.014

8. Wen WS, Yuan ZM, Ma SJ, Xu J, Yuan DT. CRISPR-Cas9 systems: versatile cancer modeling platforms and promising therapeutic strategies. Int J Cancer. 2016;138(6):1328-1336. doi:10.1002/ijc.29626

9. Bhattacharjee R, Purkayastha KD, Adapa D, Choudhury A. CRISPR/ Cas9 genome editing system in the diagnosis and treatment of cancer. J RNAi Gene Silencing. 2017;13:585-591.

10. Gwiazda KS, Grier AE, Sahni J, et al. High-efficiency CRISPR/Cas9mediated gene editing in primary human T-cells using mutant adenoviral E4orf6/E1b55k "helper" proteins. Mol Ther. 2016;24 (9):1570-1580. doi:10.1038/mt.2016.105

11. Wang T, Wei JJ, Sabatini DM, Lander ES. Genetic screens in human cells using the CRISPR-Cas9 system. Science. 2014;343 (6166):80-84. doi:10.1126/science.1246981

12. Ran FA, Cong L, Yan WX, et al. In vivo genome editing using Staphylococcus aureus Cas9. Nature. 2015;520(7546):186-191. doi:10.1038/nature14299

13. Gori JL, Hsu PD, Maeder ML, Shen S, Welstead GG, Bumcrot D. Delivery and specificity of CRISPR/Cas 9 genome editing technologies for human gene therapy. Hum Gene Ther. 2015;26(7):443-451. doi:10.1089/hum.2015.074

14. Shinmyo Y, Tanaka S, Tsunoda S, Hosomichi K, Tajima A, Kawasaki H. CRISPR/Cas9-mediated gene knockout in the mouse brain using in utero electroporation. Sci Rep. 2016;6(1):1-3. doi:10.1038/srep20611
15. Ratan ZA, Son YJ, Haidere MF, et al. CRISPR-Cas9: a promising genetic engineering approach in cancer research. Ther Adv Med Oncol. 2018;10:1758834018755089. doi:10.1177/1758834018755089

16. Jamal M, Ullah A, Ahsan M, et al. Treating genetic disorders using state-of-the-art technology. Curr Issues Mol Biol. 2017;26:33-46. doi: $10.21775 / \mathrm{cimb} .026 .033$

17. Kim EJ, Kang KH, Ju JH. CRISPR-Cas9: a promising tool for gene editing on induced pluripotent stem cells. Korean J Intern Med. 2017;32(1):42. doi:10.3904/kjim.2016.198

18. Liu C, Zhang L, Liu H, Cheng K. Delivery strategies of the CRISPR-Cas9 gene-editing system for therapeutic applications. $J$ Control Release. 2017;266:17-26. doi:10.1016/j.jconrel.2017.09.012

19. Sánchez-Rivera FJ, Jacks T. Applications of the CRISPR-Cas9 system in cancer biology. Nat Rev Cancer. 2015;15(7):387-393. doi: $10.1038 / \mathrm{nrc} 3950$

20. Lino CA, Harper JC, Carney JP, Timlin JA. Delivering CRISPR: a review of the challenges and approaches. Drug Deliv. 2018;25 (1):1234-1257. doi:10.1080/10717544.2018.1474964

21. McGinn J, Marraffini LA. Molecular mechanisms of CRISPR-Cas spacer acquisition. Nat Rev Microbiol. 2019;17(1):7-12. doi:10.1038/ s41579-018-0071-7

22. Finn JD, Smith AR, Patel MC, et al. A single administration of CRISPR/ Cas9 lipid nanoparticles achieves robust and persistent in vivo genome editing. Cell Rep. 2018;22(9):2227-2235. doi:10.1016/j. celrep.2018.02.014

23. Martinez-Lage M, Puig-Serra P, Menendez P, Torres-Ruiz R, RodriguezPerales S. CRISPR/Cas9 for cancer therapy: hopes and challenges. Biomedicines. 2018;6(4):105. doi:10.3390/biomedicines6040105

24. Kolli N, Lu M, Maiti P, Rossignol J, Dunbar GL. Application of the gene-editing tool, CRISPR-Cas9, for treating neurodegenerative diseases. Neurochem Int. 2018;112:187-196. doi:10.1016/j. neuint.2017.07.007

25. Lu Y. PD-1 knockout engineered T cells for metastatic non-small cell lung cancer. ClinicalTrials.gov. 2019. doi:10.1016/j.compcom.2006.05.002

26. Doudna JA. The promise and challenge of therapeutic genome editing. Nature. 2020;578(7794):229-236. doi:10.1038/s41586-020-19 78-5

27. Pandey VK, Tripathi A, Bhushan R, Ali A, Dubey PK, Therapy G. Application of CRISPR/Cas9 genome editing in genetic disorders: a systematic review up to date. J Genet Syndr Gene Ther. 2017;8(2). doi:10.4172/2157-7412.1000321

28. Jiang C, Lin X, Zhao Z. Applications of CRISPR/Cas9 technology in the treatment of lung cancer. Trends Mol Med. 2019;25 (11):1039-1049. doi:10.1016/j.molmed.2019.07.007

29. Tang H, Shrager JB. CRISPR/Cas-mediated genome editing to treat EGFR-mutant lung cancer: a personalized molecular surgical therapy. EMBO Mol Med. 2016;8(2):83-85. doi:10.15252/emmm.201506 006

30. Lizotte PH, Hong RL, Luster TA, et al. A high-throughput immune-oncology screen identifies EGFR inhibitors as potent enhancers of antigen-specific cytotoxic T-lymphocyte tumor cell killing. Cancer Immunol Res. 2018;6(12):1511-1523. doi:10.1158/23266066.CIR-18-0193

31. Zhao Z, Shi L, Zhang W, et al. CRISPR knock out of programmed cell death protein 1 enhances the anti-tumor activity of cytotoxic $\mathrm{T}$ lymphocytes. Oncotarget. 2018;9(4):5208. doi:10.18632/ oncotarget. 23730

32. Zhang B. CRISPR/Cas gene therapy. J Cell Physiol. 2021;236 (4):2459-2481.

33. Lu Y, Xue J, Deng T, et al. Safety and feasibility of CRISPR-edited $\mathrm{T}$ cells in patients with refractory non-small-cell lung cancer. Nat Med. 2020;26(5):732-740. doi:10.1038/s41591-020-0840-5

34. Li H, Yang Y, Hong W, Huang M, Wu M, Zhao X. Applications of genome editing technology in the targeted therapy of human diseases: mechanisms, advances, and prospects. Signal Transduct Target Ther. 2020;5(1):1-23. 
35. Xu M, Weng Q, Ji J. Applications and advances of CRISPR/Cas9 in an animal cancer model. Brief Funct Genomics. 2020;19(3):235-241. doi:10.1093/bfgp/elaa002

36. Ma CC, Wang ZL, Xu T, He ZY, Wei YQ. The approved gene therapy drugs worldwide: from 1998 to 2019. Biotechnol Adv. 2020;40:107502. doi:10.1016/j.biotechadv.2019.107502

37. Hanna RE, Doench JG. Design and analysis of CRISPR-Cas experiments. Nat Biotechnol. 2020;38(7):813-823. doi:10.1038/s41587020-0490-7
38. Hong W, Huang M, Wei Y, Wei X. A new and promising application of gene editing: CRISPR-controlled smart materials for tissue engineering, bioelectronics, and diagnostics. Sci China Life Sci. 2019;62 (11):1547-1549. doi:10.1007/s11427-019-1576-0

\section{Publish your work in this journal}

Biologics: Targets and Therapy is an international, peer-reviewed journal focusing on the patho-physiological rationale for and clinical application of Biologic agents in the management of autoimmune diseases, cancers or other pathologies where a molecular target can be identified. This journal is indexed on PubMed Central, CAS, EMBase,
Scopus and the Elsevier Bibliographic databases. The manuscript management system is completely online and includes a very quick and fair peer-review system, which is all easy to use. Visit http://www.dovepress.com/testimonials.php to read real quotes from published authors. 